Edwin H. G. Oei

Jeroen J. Nikken

Abida Z. Ginai

Gabriel P. Krestin

Jan A. N. Verhaar

Arie B. van Vugt

M. G. Myriam Hunink

From the Program for the

Assessment of Radiological

Technology (ART Program)

\title{
Costs and effectiveness of a brief MRI examination of patients with acute knee injury
}

Present address:

E. H. G. Oei · A. Z. Ginai •

G. P. Krestin

Department of Radiology,

Erasmus MC, University Medical

Center Rotterdam,

s-Gravendijkwal 230,

3015 CE Rotterdam, The Netherlands

This study was supported in part by the Revolving Fund from the Erasmus University Medical Center Rotterdam and by an unrestricted grant from Esaote S.p.A., Genoa, Italy.

E. H. G. Oei · J. J. Nikken •

A. Z. Ginai - G. P. Krestin ·

M. G. M. Hunink

Department of Radiology,

Erasmus MC,

University Medical Center Rotterdam,

Dr. Molewaterplein 50,

3015 GE Rotterdam, The Netherlands

E. H. G. Oei · J. J. Nikken •

M. G. M. Hunink $(\bowtie)$

Department of Epidemiology and

Biostatistics, Erasmus MC,

University Medical Center Rotterdam,

Dr. Molewaterplein 50,

3015 GE Rotterdam, The Netherlands

e-mail: m.hunink@erasmusmc.nl

Tel.: +31-10-7043391

Fax: +31-10-7044657

\section{J. A. N. Verhaar}

Department of Orthopaedic Surgery,

Erasmus MC, University Medical

Center Rotterdam,

Dr. Molewaterplein 50,

3015 GE Rotterdam, The Netherlands

\section{A. B. van Vugt}

Department of Traumatology,

Erasmus MC, University Medical

Center Rotterdam,

Dr. Molewaterplein 50,

3015 GE Rotterdam, The Netherlands
Present address:

J. J. Nikken

Department of Radiology, Sint

Franciscus Gasthuis, Kleiweg 500,

3045 PM Rotterdam, The Netherlands

Present address:

J. A. N. Verhaar

Department of Orthopaedic Surgery,

Erasmus MC, University Medical

Center Rotterdam,

s-Gravendijkwal 230,

3015 CE Rotterdam, The Netherlands

Present address:

A. B. van Vugt

Department of Traumatology,

UMC St Radboud,

P.O. Box 9101, 6500

HB Nijmegen, The Netherlands

\begin{abstract}
The aim of this study was to assess the costs and effectiveness of selective short magnetic resonance imaging (MRI) in patients with acute knee injury. A model was developed to evaluate the selective use of MRI in patients with acute knee injury and no fracture on radiography based on the results of a trial in which 208 patients were randomized between radiography only and radiography plus MRI. We analyzed medical (diagnostic and therapeutic) costs, quality of life,
\end{abstract}

duration of diagnostic workup, number of additional diagnostic examinations, time absent from work, and time to convalescence during a 6-month follow-up period. Quality of life was lowest (EuroQol at 6 weeks 0.61 (95\% CI 0.54-0.67)); duration of diagnostic workup, absence from work, and time to convalescence were longest; and the number of diagnostic examinations was largest with radiography only. These outcomes were more favorable for both MRI strategies (EuroQol at 6 weeks 0.72 (95\% CI $0.67-0.77$ ) for both). Mean total costs were 2,593 euros (95\% CI 1,8153,372 ) with radiography only, 2,116 euros (95\% CI 1,488-2,743) with radiography plus MRI, and 1,973 euros (95\% CI 1,401-2,543) with selective MRI. The results suggest that selective use of a short MRI examination saves costs and potentially increases effectiveness in patients with acute knee injury without a fracture on radiography.

Keywords Magnetic resonance imaging $\cdot$ Knee $\cdot$ Cost-effectiveness · Trauma 


\section{Introduction}

Magnetic resonance imaging (MRI) is an established imaging tool in the evaluation of acute knee injuries. The main purpose of MRI for knee trauma is to determine if therapeutic arthroscopy is indicated, usually in the case of persisting symptoms. This application of MRI has been studied extensively [1-5]. MRI is, however, rarely performed at initial presentation of a patient with an acute knee trauma, mainly because of the generally high costs, long examination duration, and limited availability in this clinical setting. Diagnostic information from MRI could be extremely valuable, however, since reliable physical examination shortly after a knee trauma is often hampered by swelling and pain [6, 7]. MRI, therefore, could play a significant role in the routine initial examination of patients with acute knee injury.

A requisite would be the easy availability of an MRI system, a relatively inexpensive MRI examination, and a short examination time. This can be achieved by using a low field dedicated extremity MRI system with a short data acquisition protocol $[8,9]$. Although the low field strength and shortening of the MRI protocol reduce image quality compared with a standard MRI examination, it has been demonstrated that lower magnetic field strength does not substantially reduce the diagnostic performance for most traumatic knee abnormalities $[1,10-12]$. The information obtained from MRI could be sufficient to reduce the time to completion of the diagnostic workup and to influence the treatment strategy. This information could influence the decision whether follow-up of the patient is warranted, whether treatment is indicated, or whether the patient can be sent home without the need for follow-up. Early detection of traumatic abnormalities could lead to earlier treatment of the patient and potentially to earlier recovery. This could lead to earlier resumption of work, resulting in a decrease of production losses, and thereby a decrease of costs to society.

To evaluate the use of MRI in this setting, we previously performed a randomized controlled trial (RCT) and costeffectiveness analysis and found that a short dedicated extremity MRI examination in addition to radiography in all patients with acute knee injury improves prediction of the need for additional treatment [8], shortens the time to completion of diagnostic workup, reduces the number of additional diagnostic procedures, improves quality of life in the first 6 weeks compared with radiography alone, and reduces societal costs associated with lost productivity (although the last of these was nonsignificant [9]). In the previously published RCT, patients underwent MRI regardless of the radiography findings, although it can be argued that MRI in the examination of cases of knee trauma has limited added value for the initial treatment if a fracture has already been demonstrated on radiography.

Therefore, the aim of the present study was to assess the costs and effectiveness of performing a short MRI examination on a low field dedicated extremity MRI system in the evaluation of acute knee trauma selectively in patients without a fracture on radiography. This was compared with MRI in all patients and radiography only as initial diagnostic strategies.

\section{Materials and methods}

A model was developed to evaluate radiography followed by selective use of a short MRI examination if radiography showed no fracture in patients with acute knee injury. The model was based on the results of an RCT [9].

\section{Study design}

We performed a prospective, pragmatic RCT in a university hospital, including patients with a traumatic knee injury, which had occurred within the preceding 7 days before presentation. All patients were examined by an emergency physician, traumatologist, or orthopaedic surgeon and patients were included if radiography of the knee was ordered. Exclusion criteria were pre-existing knee complaints; compound fracture; substantial injury of the head, back, thorax, or abdomen; need for urgent treatment; and intoxication. The subjects were randomized between two diagnostic strategies consisting of plain radiography alone (strategy 1 , reference strategy) and radiography followed by a short MRI examination (strategy 2). Since this was not one of the study arms of the RCT, we modeled a third strategy consisting of plain radiography, followed by a selective short MRI examination, only if no fracture was visible on the radiograph. Modeling was performed by using a composite of the results of patients from strategy 1 (no MRI examination) who showed a fracture on the radiograph, and patients from strategy 2 (radiography followed by MRI) who did not show a fracture on the radiograph. In this way the costs and effects of the third strategy could be obtained from the randomized patient groups.

Patients were randomized by drawing from consecutively numbered, sealed envelopes containing computergenerated random assignments. Block randomization was used with a block size of 20 to obtain equal numbers of patients in both strategies. Research staff and radiology technologists on service carried out the inclusion from 0800 to 2300 hours, 7 days a week. All patients were given written and oral information about the goal of the study, and all participating subjects gave informed consent. The study was approved by the institutional review board of Erasmus MC, University Medical Center, Rotterdam, The Netherlands. The results of the RCT are reported in accordance with the CONSORT statement [13].

Imaging technique and interpretation

All patients underwent anteroposterior and lateral radiography of the affected knee, and additional patellar or tunnel 
views if considered necessary. MRI was performed immediately following radiography using a 0.2 -T dedicated extremity MRI system (Artoscan M, Esaote S.p.a., Genoa, Italy) with an MR protocol as described previously $[8,9]$. The average duration of MR data acquisition was 6 min and the total MR examination time, including MRI start-up and patient positioning was on average $15 \mathrm{~min}$. The MRI was assessed by an experienced musculoskeletal radiologist (A.Z.G., 25 years of experience) or by a resident on service during evenings and weekends. The residents were in their second to fifth year of training. The result was reported to the treating physician immediately. Resident interpretations were re-read the next working day by the experienced musculoskeletal radiologist. In the case of a different interpretation the physician was informed.

\section{Follow-up}

Data were collected on utilization of medical resources, quality of life, and production losses caused by absence from work and time to convalescence. The follow-up period was 6 months. Although 6 months is relatively short for outcome assessment, we expected that this period would be long enough for relevant differences across the strategies to emerge. The effect of the availability of more diagnostic information in the initial stage is not likely to cause a significant difference in costs beyond 6 months after trauma. Questionnaires were mailed to all subjects 1 week, 6 weeks, 3 months, and 6 months after inclusion. These questionnaires included quality of life measuring instruments as well as questions about utilization of medical resources outside our hospital, out-of-pocket expenses, days off work, and time to convalescence. If questionnaires were not returned we interviewed the patient by telephone and urged them to return the quality of life questionnaires. In addition to the information from the questionnaires, data were obtained from patient records and from the computerized hospital information system.

\section{Measurement of effectiveness}

Quality of life was measured using the EuroQol $[14,15]$ and the Short Form 36 Health Survey (SF-36) [16]. Using the EuroQol it is possible to assign one preference-based score, which can be calculated using a regression equation [17]. The SF-36 consists of 36 questions covering eight domains (physical functioning, role-physical, bodily pain, general health, vitality, social functioning, role-emotional, and mental health). An algorithm is used to assign values to each domain.

From the moment a patient indicated on one of the four questionnaires that he or she did not have complaints of the injured knee anymore, we assumed that no further change in quality of life related to the initial trauma would occur, and the EuroQol and SF-36 scores measured at that point in time were extrapolated to the remaining questionnaires. If a person indicated that he/she did not have complaints anymore but did not fill in the quality of life questionnaires, mean values of all other complaint-free patients from the same randomized group were used, since it is well known that imputation of missing values results in less bias than analysis of complete cases only [18, 19]. We used linear regression analysis to analyze if these mean values were influenced by age and sex and adjusted accordingly.

The time to completion of the diagnostic workup was defined as the time from initial presentation to the last diagnostic examination. The number of additional diagnostic procedures was assessed by reviewing the hospital information system and by information from the questionnaires or telephone inquiry if the examinations had been performed outside our hospital. The number of days absent from work and the time to recovery were obtained from the questionnaires or, if the questionnaires were not returned, by telephone inquiry. A patient was regarded to have recovered if he or she did not have daily complaints anymore.

\section{Measurement of costs}

All costs relevant from the societal perspective, including medical and nonmedical costs, were recorded during a follow-up period of 6 months. Medical costs consisted of costs of diagnostic procedures and treatment both inside and outside the hospital as well as patient travel costs. Costs of initial treatment as well as diagnostic procedures were calculated using a bottom-up approach [20], taking into account the initial investment of equipment, additional costs during use, maintenance, years of use, discounting and annuitization [21], number of procedures per year, personnel costs, materials used, room rent, housekeeping, administration, and overhead costs. Costs were discounted at a rate of 3\% per annum [22]. Estimated actual costs of hospital visits and hospital admissions were obtained from the Dutch Council for Care Insurance [20]. For costs of operations we used reimbursement tariffs as established by the Dutch Central Organ for Tariffs in Healthcare.

Out-of-pocket expenses and patient time costs were recorded as direct nonmedical costs. Patient time costs were included in the cost analysis [22] in order to capture the gain in effectiveness obtained through a more expedient diagnostic workup in monetary units. Patient time costs were determined by multiplying the time spent on followup, diagnostic and therapeutic procedures (including travel time, and waiting time) by the average net income of subjects stratified for age (obtained from the Dutch Central Bureau for Statistics, data for both working and nonworking subjects in the Netherlands adjusted for the year 2007).

Costs associated with production losses were estimated using the 'friction cost method' as described by Koopmanschap et al. and recommended in guidelines for 
cost-effectiveness analysis [22, 23]. According to this method costs to society are only generated during the time it takes to replace a sick employee, which is called the friction period. After the friction period no costs to society are generated, since the sick employee is replaced by someone drawn from the ranks of the unemployed. For short-term absence from work the loss of productivity may be low, because work may be performed by colleagues or work can be postponed and performed on return of the sick employee. For long-term or permanent absence extra costs of maintaining production, costs of production loss, costs of filling the vacancy, and costs of training the new employee are incorporated.

The costs of lost productivity per day absent from work were calculated using friction cost data estimated for working people in the Netherlands in 1998, based on sex and age [20], adjusted for the year 2007. The friction period for the year 1998 was estimated to be 4 months [20], but because of increased shortage on the labor market we estimated the friction period in 2007 to be 6 months (personal communication, Koopmanschap). Costs were analyzed on an intention-to-diagnose-and-treat basis.

The effect of uncertainty of the involved parameters on the robustness of our results was studied in a sensitivity
Table 1 Baseline characteristics of the patient groups in the three strategies

\begin{tabular}{llll}
\hline & $\begin{array}{l}\text { Strategy 1 } \\
(n=93)\end{array}$ & $\begin{array}{l}\text { Strategy 2 } \\
(n=96)\end{array}$ & $\begin{array}{l}\text { Strategy 3 } \\
(n=91)\end{array}$ \\
\hline $\begin{array}{l}\text { Mean age (years) } \\
\text { Sex (percentage male) }\end{array}$ & 34.7 & 32.2 & 33.7 \\
$\begin{array}{l}\text { Fracture visible on X-ray } \\
\text { (number of patients) }\end{array}$ & 8 & 62 & 63 \\
$\begin{array}{c}\text { Type of trauma } \\
\quad \text { direct/indirect/unknown) }\end{array}$ & $41 / 51 / 1$ & $36 / 59 / 1$ & $36 / 55 / 0$ \\
\hline
\end{tabular}

Strategy 1: only radiography

Strategy 2: radiography plus MRI

Strategy 3: MRI if no fracture on radiography

analysis. One-way sensitivity analysis was conducted by exploring a range from 50 to $200 \%$ for each parameter. Parameters that appeared sensitive in the one-way analysis were analyzed in a three-way sensitivity analysis.

The analyses were performed using Microsoft Excel 97 SR-2 (Microsoft Corp, Redmond, WA) and SPSS for Windows (release 10.0.0; SPSS Inc., Chicago, IL, USA) software packages. Because one of our strategies is a composite of the results of the other two, statistical testing

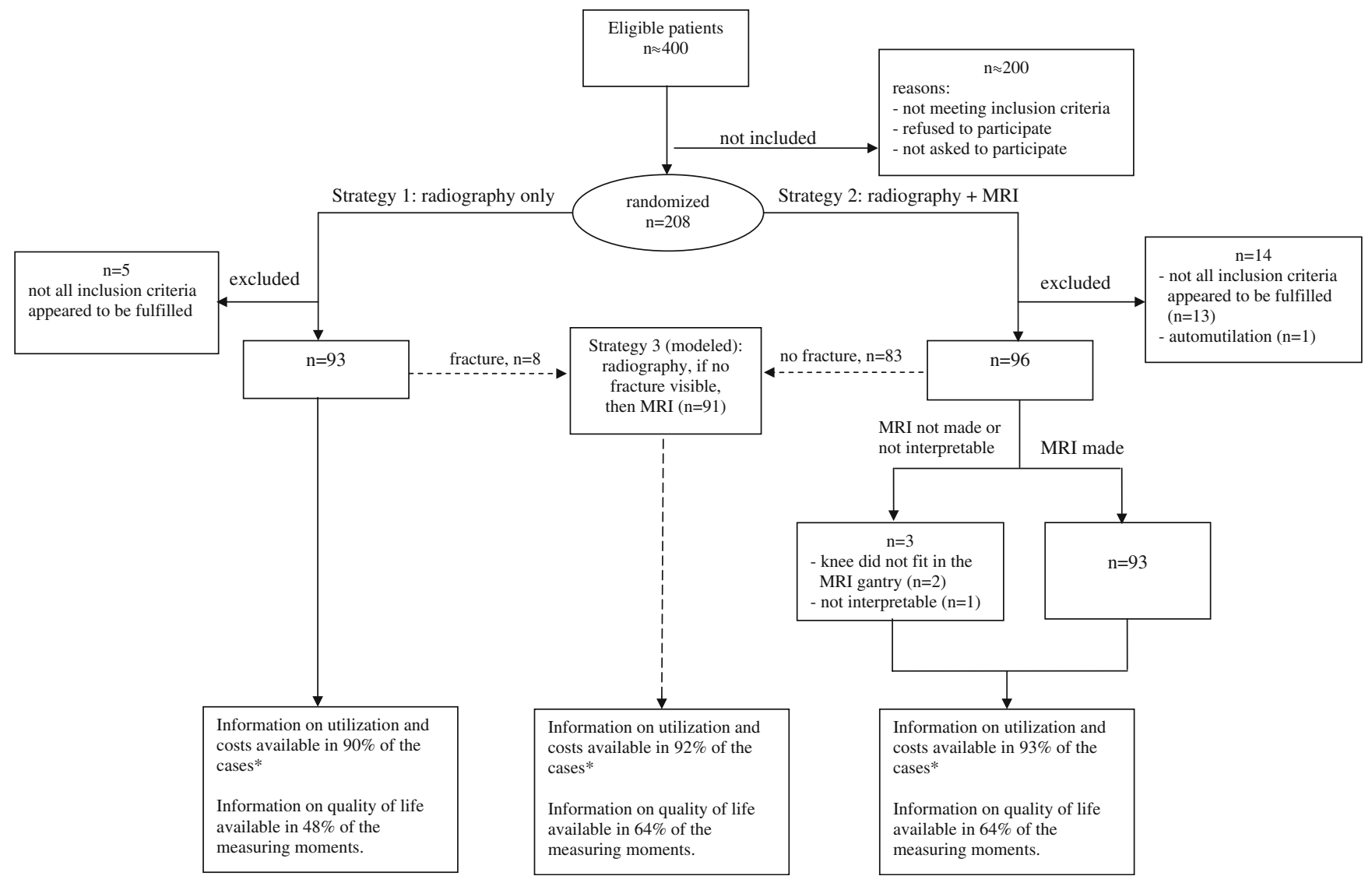

Fig. 1 Flow diagram of subjects passing through the trial. *The remaining percentage concerns patients that were not treated in our hospital, but information on possible treatment elsewhere is lacking 
Table 2 Final diagnoses in 189 patients with acute knee injury (one patient may have more than one diagnosis)

\begin{tabular}{|c|c|c|c|}
\hline & Strategy 1 & Strategy 2 & Strategy 3 \\
\hline No abnormalities & 0 & 2 & 2 \\
\hline Contusion & 18 & 24 & 25 \\
\hline Distortion $^{\mathrm{a}}$ & 21 & 14 & 14 \\
\hline \multicolumn{4}{|l|}{ Fracture } \\
\hline Patella & 4 & 6 & 4 \\
\hline Tibial plateau & 0 & 2 & 0 \\
\hline Fibula head avulsion & 0 & 1 & 0 \\
\hline Avulsion at the MCL origin & 0 & 1 & 0 \\
\hline Segond fracture & 1 & 0 & 1 \\
\hline Osteochondral fracture & 3 & 3 & 3 \\
\hline Quadriceps tendon rupture & 1 & 0 & 0 \\
\hline Medial meniscus tear & 8 & 11 & 11 \\
\hline Lateral meniscus tear & 4 & 5 & 4 \\
\hline \multicolumn{4}{|l|}{ Cruciate ligament rupture } \\
\hline ACL partial & 5 & 3 & 3 \\
\hline ACL total & 7 & 14 & 12 \\
\hline PCL partial & 0 & 1 & 1 \\
\hline PCL total & 1 & 0 & 0 \\
\hline ACL+PCL total & 1 & 0 & 0 \\
\hline ACL+PCL partial & 1 & 0 & 0 \\
\hline \multicolumn{4}{|l|}{ Collateral ligament rupture } \\
\hline MCL partial & 10 & 6 & 6 \\
\hline MCL total & 3 & 4 & 3 \\
\hline LCL partial & 0 & 1 & 1 \\
\hline LCL total & 0 & 0 & 0 \\
\hline Prepatellar bursitis & 0 & 1 & 1 \\
\hline Traumatized gonarthrosis & 3 & 1 & 1 \\
\hline Unknown $^{\mathrm{b}}$ & 9 & 5 & 5 \\
\hline
\end{tabular}

Strategy 1: only radiography

Strategy 2: radiography plus MRI

Strategy 3: MRI if no fracture on radiography

$A C L$ anterior cruciate ligament, $P C L$ posterior cruciate ligament,

$M C L$ medial collateral ligament, $L C L$ lateral collateral ligament

${ }^{a} A$ distortion was defined as an indirect trauma (torsion, hyperextension, varus, or valgus) without signs of osseous, meniscal, or ligamentous injury during initial evaluation or follow-up

${ }^{b}$ Final diagnosis could not be established (e.g., patient had persistent pain, but did not seek medical attention) for differences was not justified. We therefore chose to report $95 \%$ confidence intervals (CIs) instead of $p$ values.

\section{Results}

From August 1999 to May 2001, 208 patients were included. A flow diagram of patients passing through the study is presented in Fig. 1. About half of the eligible patients were randomized: a few patients refused to participate, but the main reason for missing potential candidates was the fact that due to the inclusion timeincluding evenings and weekends - many radiology technologists were involved in asking the patients to participate, which was forgotten regularly. On review, 18 patients did not fulfill all inclusion criteria and one case was suspected of automutilation; these 19 patients were excluded from the analysis. Of the remaining 189 patients 96 patients were allocated to the MRI strategy and 93 patients to the reference strategy (i.e., strategy 1, only radiography). In two patients allocated to the MRI strategy, the MRI could not be made because the knee could not be positioned in the center of the magnet bore: one patient had a locked knee, and in one case the knee was too large to fit into the magnet. In one very obese patient the MRI was uninterpretable. In accordance with the intention-to-diagnose-and-treat principle, these three patients remained in the MRI strategy in the analyses. The baseline characteristics are described in Table 1. Eight patients in strategy 1 (only radiography) showed a fracture on the radiograph, and 83 patients in strategy 2 (radiography plus MRI) showed no fracture on the radiograph. These 91 patients formed the subject group for strategy 3 (MRI if no fracture on radiography). The final diagnoses as assessed using all information including follow-up are listed in Table 2.

\section{Effectiveness}

Linear regression analysis showed that the mean EuroQol and SF-36-domain scores of patients without complaints were not significantly influenced by age or sex, and therefore mean values of EuroQol and SF-36-domain values were used for
Fig. 2 Mean EuroQol scores and $95 \%$ confidence intervals for the three strategies measured at four points in time after the initial injury

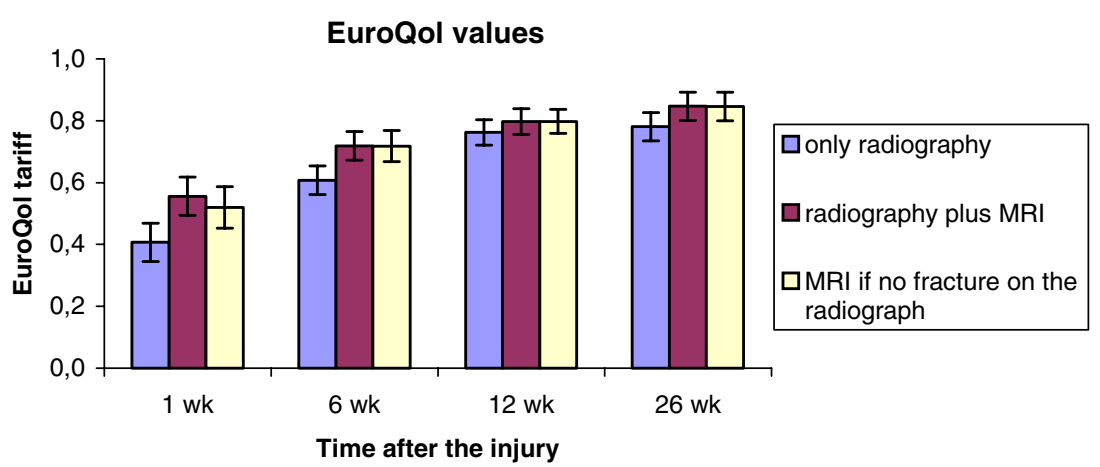



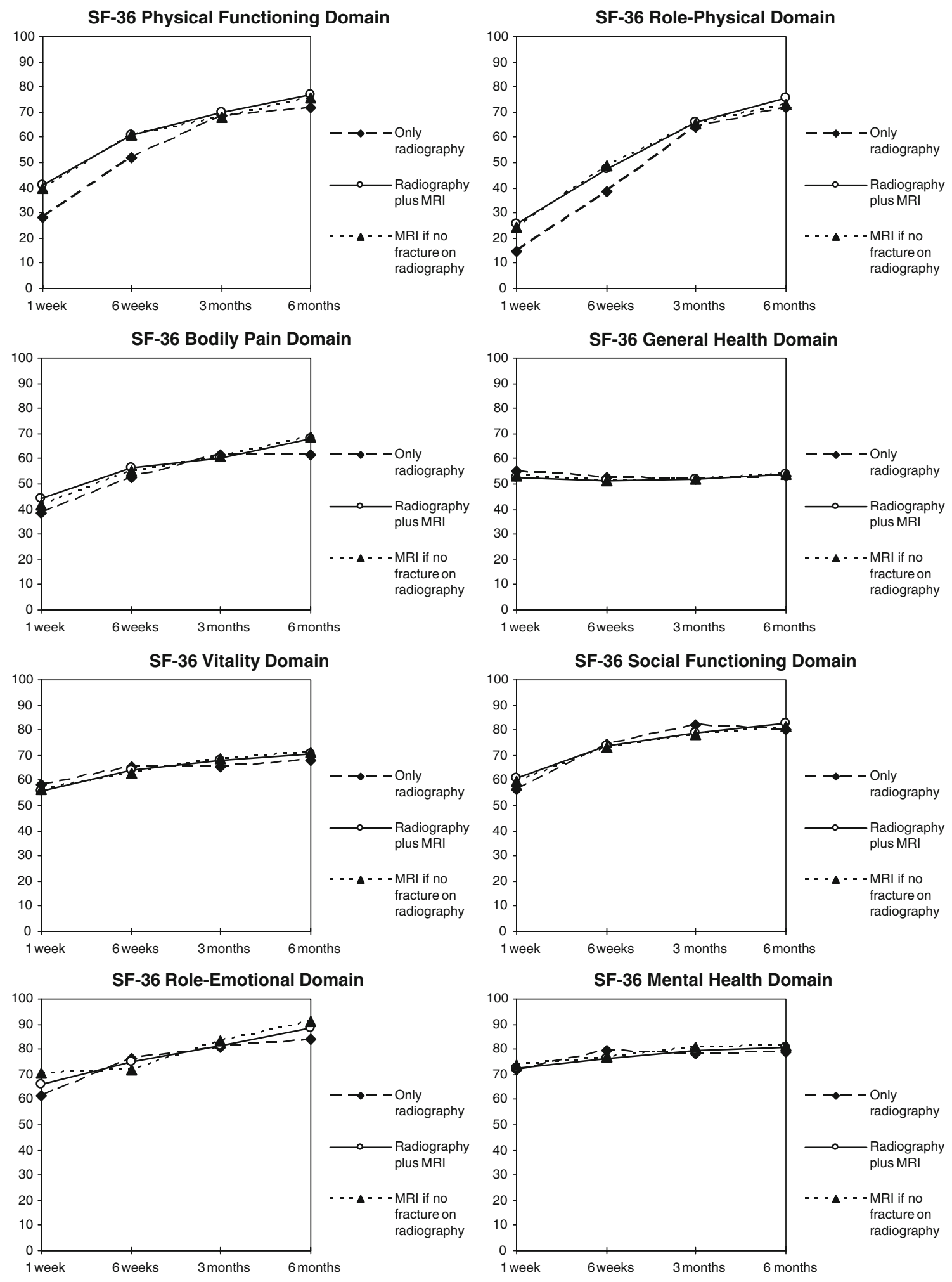

Fig. 3 Mean SF-36 scores per domain for the three strategies at 1-week, 6-weeks, 3-months, and 6-months follow-up. A higher score implies a more favorable outcome 
Table 3 Effectiveness results

\begin{tabular}{llll}
\hline & Strategy 1 $(n=93)$ & Strategy 2 $(n=96)$ & Strategy 3 $(n=91)$ \\
\hline Mean time to last diagnostic procedure (days) & $17.3(9.3-25.2)$ & $3.5(0.0-7.6)$ & $2.0(0.0-4.1)$ \\
Number of additional diagnostic procedures & 35 & 9 & 10 \\
Mean duration of absence from work (days) & $12.4(7.5-17.3)$ & $9.6(5.9-13.2)$ & $8.6(5.0-12.3)$ \\
Mean time to convalescence (days) & $76.6(54.6-98.5)$ & $66.2(50.2-82.3)$ & $60.4(44.8-76.0)$ \\
\hline
\end{tabular}

95\% confidence intervals are enclosed in parentheses

Strategy 1: only radiography

Strategy 2: radiography plus MRI

Strategy 3: MRI if no fracture on radiography

imputation. The mean EuroQol scores per strategy are presented in Fig. 2. Patients in strategy 2 (radiography plus MRI in all patients) showed a higher EuroQol score at 1 and 6 weeks after the injury (EuroQol score 0.56 (95\% CI 0.49 0.62 ) and 0.72 (95\% CI $0.67-0.77)$, respectively) than patients in strategy 1 (only radiography; EuroQol score 0.41 (95\% CI $0.33-0.48$ ) and 0.61 (95\% CI $0.54-0.67)$, respectively). In strategy 3 (MRI if no fracture visible on the radiograph) the EuroQol score at 1 week was slightly lower but similar (EuroQol score 0.52 (95\% CI 0.45-0.59)) compared with strategy 2 (always MRI), whereas during follow-up the EuroQol scores of these two strategies were equivalent (EuroQol scores for strategies 2 and 3 were $0.72(95 \% \mathrm{CI}$ $0.67-0.77), 0.80$ (95\% CI 0.76-0.84), and 0.85 (95\% CI 0.800.89 ) at 6 weeks, 3 months, and 6 months, respectively).

All the SF-36 domains, except for general health, demonstrated an increase in score over time (Fig. 3). The two MRI strategies demonstrated similar SF-36 scores across all domains at all points in time. After 1 and 6 weeks physical functioning and physical role functioning were higher in the MRI strategies compared with radiography alone. The scores of all the domains were very similar across the three strategies at all points in time.

The time to completion of the diagnostic workup, the duration of absence from work, and the time to convalescence were shortest in strategy 3 (Table 3 ). The number of additional procedures was almost the same in the two strategies with MRI, which was considerably shorter than in the strategy with only radiography.
Costs

Medical costs were highest in the strategy with MRI in all patients (strategy 2) and lowest if no MRI was performed at all (strategy 1) (Table 4). Travel costs were relatively low and similar across the three strategies. The friction costs constituted the largest cost factor in all strategies. Total costs were lowest if MRI was used selectively in patients without a fracture on the radiograph (strategy 3), mainly owing to a reduction in friction costs. Total costs were highest in the strategy with only radiography, which again was mainly attributable to high friction costs.

\section{Sensitivity analysis}

In a one-way sensitivity analysis the cost difference between the strategies including MRI (strategies 2 and 3) and the strategy with only radiography (strategy 1) was sensitive to the estimated friction costs per friction period, to the friction period itself, and to the costs of the short MRI examination (Table 5). Three-way sensitivity analysis of friction costs, friction period, and costs of the short MRI examination (Fig. 4) demonstrated that within plausible ranges of these variables the strategies that included MRI generated less costs than the strategy with only radiography. This conclusion was even more robust for strategy 3 (MRI if no fracture on radiography) (Fig. 4b) than for strategy 2 (MRI in all patients) (Fig. 4a).

Table 4 Mean costs associated with the initial knee injury

\begin{tabular}{llll}
\hline Cost & Strategy 1 & Strategy 2 & Strategy 3 \\
\hline Medical & $704(537-873)$ & $803(565-1,043)$ & $744(604-885)$ \\
Travel & $19(16-22)$ & $20(17-23)$ & $21(17-23)$ \\
Friction & $1,755(1,042-2,468)$ & $1,154(646-1,662)$ & $1,097(585-1,610)$ \\
Time & $115(67-163)$ & $137(34-242)$ & $109(66-153)$ \\
Total & $2,593(1,815-3,372)$ & $2,116(1,488-2,743)$ & $1,973(1,401-2,543)$ \\
\hline
\end{tabular}

Costs are in euros (adjusted to the year 2007 using consumer price indices); 95\% confidence intervals are enclosed in parentheses

Strategy 1: only radiography

Strategy 2: radiography plus MRI

Strategy 3: MRI if no fracture on radiography 
Table 5 One-way sensitivity analysis of the mean total cost difference between strategies

\begin{tabular}{|c|c|c|c|c|c|c|}
\hline \multirow[b]{2}{*}{ Value of variable } & \multicolumn{3}{|c|}{ Cost-savings with strategy 2 compared with strategy 1} & \multicolumn{3}{|c|}{ Cost-savings with strategy 3 compared with strategy 1} \\
\hline & $50 \%$ & $200 \%$ & Range & $50 \%$ & $200 \%$ & Range \\
\hline Baseline cost difference & & & & & & \\
\hline Friction costs & 178 & 1080 & 902 & 293 & 1277 & 984 \\
\hline Friction period & 307 & 479 & 172 & 432 & 621 & 189 \\
\hline Short MRI costs & 511 & 413 & 98 & 651 & 561 & 90 \\
\hline Outpatient visit costs & 494 & 448 & 46 & 635 & 594 & 41 \\
\hline Time costs & 489 & 456 & 33 & 618 & 627 & 9 \\
\hline Costs per hospital day & 486 & 463 & 23 & 601 & 661 & 60 \\
\hline Physiotherapy costs & 484 & 467 & 17 & 634 & 597 & 37 \\
\hline Standard MRI costs & 474 & 486 & 12 & 610 & 643 & 33 \\
\hline Operative therapy costs & 477 & 482 & 5 & 617 & 629 & 12 \\
\hline Radiography costs & 480 & 474 & 6 & 622 & 620 & 2 \\
\hline
\end{tabular}

Cost-savings using strategy 2 and 3, respectively, compared with strategy 1 (reference strategy) for 50\% and $200 \%$ of the variable value are presented. Only the most sensitive variables are presented. Costs are in euros

Strategy 1: only radiography

Strategy 2: radiography plus MRI

Strategy 3: MRI if no fracture on radiography

\section{Discussion}

MRI is seldom used as an initial diagnostic tool in acute knee injury because of the perceived high costs and long duration of the examination. We studied the costs and effectiveness of applying a short MRI examination in this setting. Two MRI strategies were analyzed and compared with the strategy of radiography alone, namely performing MRI in all patients and selective use of MRI if no fracture is demonstrated on the radiograph. The rationale behind the latter strategy is that we considered it plausible that the addition of MRI to radiography in patients who already show a fracture on the radiograph has limited value for the (initial) treatment: in most cases treatment will be determined by the fracture. This strategy was modeled, since it was not incorporated into the trial. Modeling created limitations in the comparison of the strategies, since differences between the modeled and the observed strategies could not be tested statistically. However, we chose to use a modeled strategy because it created the opportunity to analyze a realistic and relevant strategy beyond the scope of the original trial.

The selective application of MRI in patients without a fracture on the radiograph resulted in a slight improvement in quality of life during the first 6 weeks compared with using radiography only. The quality of life was almost identical for the two MRI strategies. Time to diagnosis, duration of absence from work, and time to convalescence were all slightly shorter compared with MRI in all patients and substantially shorter compared with only radiography.

Whilst performing this study we assumed that the early diagnostic information from MRI would result in a more appropriate and earlier treatment. Although this is true for many cases, we acknowledge that there is a potential danger of overtreatment. Some meniscal tears may heal or become
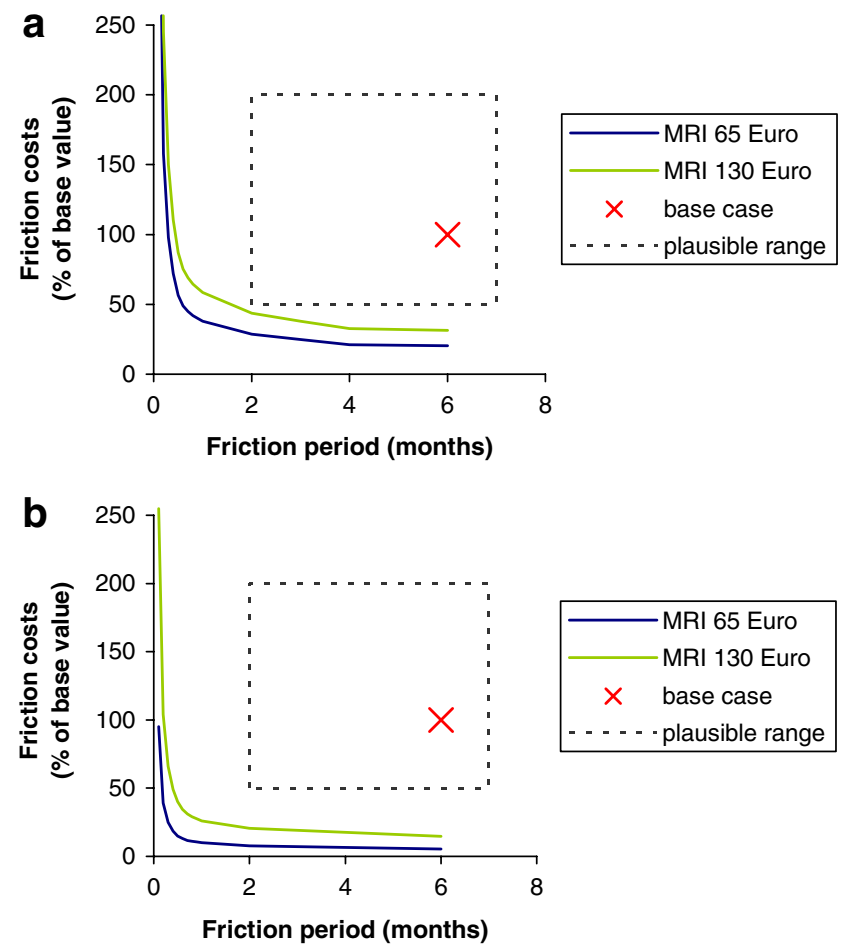

Fig. 4 Three-way sensitivity analysis on friction costs and friction period for the base value of the short MRI examination (65 euros) and twice this base value. Radiography and MRI in all patients compared with radiography only (a); radiography in all patients followed by MRI if no fracture is visible on the radiograph compared with radiography only (b). In the area above the threshold lines the strategy that includes MRI generates less costs than the reference strategy. In the area below the threshold lines the strategy including MRI generates more costs than the reference strategy. A plausible range of friction costs and friction period is indicated 
symptomless without surgery [24]. Still, if the short MRI examination leads to an overall reduction in costs without a reduction in quality of life as was demonstrated, the benefits of more accurate early diagnostic information outweighs the risk of overtreatment. On the other hand, we acknowledge that MRI should not be regarded as a substitute for thorough clinical history taking and examination, and that MRI may be unnecessary in certain patients. If there are obvious mechanical symptoms such as locking, arthroscopy is required regardless of MRI findings $[25,26]$. In less equivocal cases, however, MRI may be useful, since physical examination is often unreliable if performed in the acute stage $[6,7]$.

Medical costs were lowest if only radiography was performed. As expected, if an MRI was performed selectively in the absence of a fracture, medical costs were lower than if MRI was performed in all patients. Thus, the application of a short MRI examination did not lead to a reduction in costs of subsequent diagnostic and therapeutic procedures. The main reduction in costs was brought about by a reduction in lost productivity, which was similar for both MRI strategies. The difference in time costs was small across strategies because the mean time spent for diagnostic and therapeutic procedures was similar. Travel costs were similar and low for all groups. This is plausible in the Dutch situation, since the density of hospitals in the Netherlands is high, and travel distance to the nearest hospital is generally short.

Our conclusions were robust in sensitivity analysis. A threeway analysis of the most sensitive parameters (friction costs, friction period, and costs of the short MRI examination) did not influence our conclusions. Our results were, however, influenced by heterogeneity of the patient populations, expressed by the wide confidence intervals of the costs (Table 4). This heterogeneity within the patient groups was to be expected since we considered all patients with traumatic knee injury, ranging from a mild injury, without need for treatment to trauma with extensive internal derangement. Friction costs were especially subject to heterogeneity in the studied population. Since a short period off work may generate considerable costs, absenteeism caused high mean friction costs in spite of the fact that only a small number of patients were absent from work.

The low sensitivity of our results to variation in costs of the MRI examination suggests that a high field MRI system can also be used for the initial evaluation of knee trauma, still with a reduction in overall costs from the societal perspective. The examination costs will increase, but the better quality images may increase reliability of the examination result, with potential improvement in patient outcome.

A limitation of the study is the fact that a considerable percentage of the potentially eligible patients were not included, although inclusion was intended to be consecutive. The major reason why patients were not randomized was because the radiology technologist on service had forgotten to ask the patient to participate. We expect that the likelihood of selection bias in these cases of nonrandomized patients is low.

In many studies the response rate to mailed questionnaires is about $60-65 \%$ [27-34]. In our study the response rate of patients that underwent both radiography and MRI was within that range, but the response rate of patients that only underwent radiography was lower, most likely because they were disappointed not to get an extra MRI examination during randomization and less willing to fill in the questionnaires. This may have biased the results on quality of life. For the data on costs we were much less dependent on the questionnaires, since we could obtain most of the data from the patient records and computerized hospital information system, sometimes complemented by telephone inquiry.

In conclusion, our results suggest that the selective use of a short MRI examination following radiography in patients with acute knee injury without a fracture on the radiograph reduces costs to society and increases effectiveness compared with a strategy of using radiography alone. The results also indicate that, compared with MRI in all patients, selective MRI in patients without a fracture on the radiograph could reduce both medical costs and costs to society without affecting health outcomes. Because the results were in part obtained from a modeling analysis they should ideally be evaluated in a prospective trial.

Acknowledgement We thank Wibeke van Leeuwen and Caroline van Bavel for their support in collecting the data.

Open Access This article is distributed under the terms of the Creative Commons Attribution Noncommercial License which permits any noncommercial use, distribution, and reproduction in any medium, provided the original author(s) and source are credited.

\section{References}

1. Oei EH, Nikken JJ, Verstijnen AC, Ginai AZ, Hunink MG (2003) MR imaging of the menisci and cruciate ligaments: a systematic review. Radiology 226:837-848
2. Elvenes J, Jerome CP, Reikeras O, Johansen O (2000) Magnetic resonance imaging as a screening procedure to avoid arthroscopy for meniscal tears. Arch Orthop Trauma Surg 120:14-16

3. Munk B, Madsen F, LundorfE et al (1998) Clinical magnetic resonance imaging and arthroscopic findings in knees: a comparative prospective study of meniscus anterior cruciate ligament and cartilage lesions. Arthroscopy 14:171-175
4. Weinstabl R, Muellner T, Vecsei V, Kainberger F, Kramer M (1997) Economic considerations for the diagnosis and therapy of meniscal lesions: can magnetic resonance imaging help reduce the expense? World J Surg 21:363-368 
5. Vincken PW, ter Braak BP, van Erkell AR et al (2002) Effectiveness of MR imaging in selection of patients for arthroscopy of the knee. Radiology 223:739-746

6. Hardaker WT Jr, Garrett WE Jr, Bassett FH 3rd (1990) Evaluation of acute traumatic hemarthrosis of the knee joint. South Med J 83:640-644

7. Mitsou A, Vallianatos P (1988) Clinical diagnosis of ruptures of the anterior cruciate ligament: a comparison between the Lachman test and the anterior drawer sign. Injury 19:427-428

8. Oei EH, Nikken JJ, Ginai AZ et al (2005) Acute knee trauma: value of a short dedicated extremity MR imaging examination for prediction of subsequent treatment. Radiology 234:125133

9. Nikken JJ, Oei EH, Ginai AZ et al (2005) Acute peripheral joint injury: cost and effectiveness of low-fieldstrength MR imaging-results of randomized controlled trial. Radiology 236:958-967

10. Vellet AD, Lee DH, Munk PL et al (1995) Anterior cruciate ligament tear: prospective evaluation of diagnostic accuracy of middle- and high-fieldstrength MR imaging at 1.5 and $0.5 \mathrm{~T}$. Radiology 197:826-830

11. Rutt BK, Lee DH (1996) The impact of field strength on image quality in MRI. J Magn Reson Imaging 6:57-62

12. Barnett MJ (1993) MR diagnosis of internal derangements of the knee: effect of field strength on efficacy. AJR Am J Roentgenol 161:115-118

13. Moher D, Schulz KF, Altman D (2001) The CONSORT statement: revised recommendations for improving the quality of reports of parallel-group randomized trials. JAMA 285:19871991

14. The EuroQol Group (1990) EuroQol-a new facility for the measurement of health-related quality of life. Health Policy 16:199-208
15. Brooks R (1996) EuroQol: the current state of play. Health Policy 37:53-72

16. Ware JE Jr, Sherbourne CD (1992) The MOS 36-item short-form health survey (SF-36). I. Conceptual framework and item selection. Med Care 30:473-483

17. Dolan P (1997) Modeling valuations for EuroQol health states. Med Care 35:1095-1108

18. Schafer J (1997) Analysis of incomplete multivariate data. Chapman \& Hall, London

19. van der Heijden GJ, Donders AR, Stijnen T, Moons KG (2006) Imputation of missing values is superior to complete case analysis and the missingindicator method in multivariable diagnostic research: a clinical example. J Clin Epidemiol 59:1102-1109

20. Oostenbrink JB, Koopmanschap MA, Rutten FFH (2000) Handleiding voor kostenonderzoek, methoden en richtlijnprijzen voor economische evaluaties in de gezondheidszorg. College voor zorgverzekeringen, Amstelveen

21. Hunink MGM, Glasziou PP (2001) Decision making in health and medicine - integrating evidence and values. Cambridge University Press, Cambridge

22. Gold MR, Siegel JE, Russell LB, Weinstein MC (1996) Costeffectiveness in health and medicine. Oxford University Press, Oxford

23. Koopmanschap MA, Rutten FF, van Ineveld BM, van Roijen L (1995) The friction cost method for measuring indirect costs of disease. J Health Econ 14:171-189

24. Solomon DH, Simel DL, Bates DW, Katz JN, Schaffer JL (2001) The rational clinical examination. Does this patient have a torn meniscus or ligament of the knee? Value of the physical examination. JAMA 286:1610-1620

25. Dandy DJ (1997) Arthroscopy and MRI for the knee. J Bone Joint Surg Br 79:520

26. Rose NE, Gold SM (1996) A comparison of accuracy between clinical examination and magnetic resonance imaging in the diagnosis of meniscal and anterior cruciate ligament tears. Arthroscopy 12:398-405
27. Kotaniemi JT, Hassi J, Kataja M et al (2001) Does non-responder bias have a significant effect on the results in a postal questionnaire study? Eur J Epidemiol 17:809-817

28. Becker A, Seitz R, Jacobi E, Leidl R (2001) (Cost evaluation by a patient questionnaire: pilot study of a weekly cost diary) Kostenmessung durch $\mathrm{Pa}-$ tientenbefragung: Pilotstudie $\mathrm{zu}$ einem Kostenwochenbuch. Rehabilitation (Stuttg) 40:12-20

29. Biering-Sorensen F, Biering-Sorensen M (2001) Sleep disturbances in the spinal cord injured: an epidemiological questionnaire investigation, including a normal population. Spinal Cord 39:505-513

30. Heath AC, Howells W, Kirk KM et al (2001) Predictors of non-response to a questionnaire survey of a volunteer twin panel: findings from the Australian 1989 twin cohort. Twin Res 4:7380

31. Bakker E, Van Gool JD, Van Sprundel M, Van Der Auwera C, Wyndaele JJ (2002) Results of a questionnaire evaluating the effects of different methods of toilet training on achieving bladder control. BJU Int 90:456-461

32. Campbell MJ, Waters WE (1990) Does anonymity increase response rate in postal questionnaire surveys about sensitive subjects? A randomised trial. J Epidemiol Community Health 44:7576

33. Lund E, Gram IT (1998) Response rate according to title and length of questionnaire. Scand J Soc Med 26:154160

34. Maconochie N, Doyle P, Davies G et al (2003) The study of reproductive outcome and the health of offspring of UK veterans of the Gulf War: methods and description of the study population. BMC Public Health 3:4 